\title{
Acceleration Modes and Transitions in Pulsed Plasma Accelerators
}

\author{
Kurt A. Polzin* and Christine M. Greve ${ }^{\dagger}$ \\ NASA-Marshall Space Flight Center, Huntsville, AL 35812
}

\begin{abstract}
I. Abstract

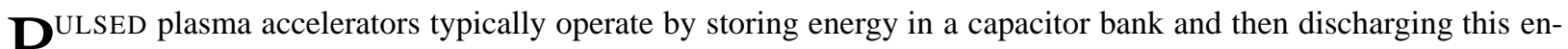
Pergy through a gas, ionizing and accelerating it through the Lorentz body force. Two plasma accelerator types employing this general scheme have typically been studied: the gas-fed pulsed plasma thruster ${ }^{1}$ and the quasi-steady magnetoplasmadynamic (MPD) accelerator. ${ }^{2}$

The gas-fed pulsed plasma accelerator is generally represented as a completely transient device discharging in $\sim 1-10 \mu \mathrm{s}$. When the capacitor bank is discharged through the gas, a current sheet forms at the breech of the thruster and propagates forward under a $\mathbf{j} \times \mathbf{B}$ body force, entraining propellant it encounters. This process is sometimes referred to as detonation-mode acceleration because the current sheet representation approximates that of a strong shock propagating through the gas. Acceleration of the initial current sheet ceases when either the current sheet reaches the end of the device and is ejected or when the current in the circuit reverses, striking a new current sheet at the breech and depriving the initial sheet of additional acceleration.

In the quasi-steady MPD accelerator, the pulse is lengthened to $\sim 1 \mathrm{~ms}$ or longer and maintained at an approximately constant level during discharge. The time over which the transient phenomena experienced during startup typically occur is short relative to the overall discharge time, which is now long enough for the plasma to assume a relatively steady-state configuration. The ionized gas flows through a stationary current channel in a manner that is sometimes referred to as the deflagration-mode of operation. The plasma experiences electromagnetic acceleration as it flows through the current channel towards the exit of the device.

A device that had a short pulse length but appeared to operate in a plasma acceleration regime different from the gas-fed pulsed plasma accelerators was developed by Cheng, et al. ${ }^{3}$ The Coaxial High ENerGy (CHENG) thruster operated on the $10 \mu$ s timescales of pulsed plasma thrusters, but claimed high thrust density, high efficiency and low electrode erosion rates, which are more consistent with the deflagration mode of acceleration.

Separate work on gas-fed pulsed plasma thrusters (PPTs) by Ziemer, et al. ${ }^{4,5}$ identified two separate regimes of performance. The regime at higher mass bits (termed Mode I in that work) possessed relatively constant thrust efficiency (ratio of jet kinetic energy to input electrical energy) as a function of mass bit. In the second regime at very low mass bits (termed Mode II), the efficiency increased with decreasing mass bit.

Work by Poehlmann et al. ${ }^{6}$ and by Sitaraman and Raja ${ }^{7}$ sought to understand the performance of the CHENG thruster and the Mode I/Mode II performance in PPTs by modeling the acceleration using the Hugoniot Relation, with the detonation and deflagration modes representing two distinct sets of solutions to the relevant conservation laws. These works studied the proposal that, depending upon the values of the various controllable parameters, the accelerator would operate in either the detonation or deflagration mode.

In the present work, we propose a variation on the explanation for the differences in performance between the various pulsed plasma accelerators. Instead of treating the accelerator as if it were only operating in one mode or the other during a pulse, we model the initial stage of the discharge in all cases as an accelerating current sheet (detonation mode). If the current sheet reaches the exit of the accelerator before the discharge is completed, the acceleration mode transitions to the deflagration mode type found in the quasi-steady MPD thrusters. This modeling method is used to demonstrate that standard gas-fed pulsed plasma accelerators, the CHENG thruster, and the quasi-steady MPD accelerator are variations of the same device, with the overall acceleration of the plasma depending upon the behavior
\end{abstract}

\footnotetext{
*Associate Fellow AIAA.

${ }^{\dagger}$ Student Member AIAA. Presently: Graduate Research Assistant, Texas A\&M University, College Station, TX.
} 
of the plasma discharge during initial transient phase and the relative lengths of the detonation and deflagration modes of operation.

\section{References}

${ }^{1}$ J. Marshall, "Performance of a Hydromagnetic Plasma Gun,” Phys. Fluids, Vol. 3, No. 1, 134-135 (1960).

${ }^{2}$ R.L. Burton, K.E. Clark, and R.G. Jahn, "Measured performance of a multimegawatt MPD thruster," J. Spacecraft Rockets, Vol. 20, No. 3 , 299-304 (1983).

${ }^{3}$ D.Y. Cheng, "Plasma deflagration and the properties of a coaxial plasma deflagration gun," Nucl. Fusion, Vol. 10, 305-317 (1970).

${ }^{4}$ J.K. Ziemer, "Performance scaling of gas-fed pulsed plasma thrusters," Ph.D. Dissertation 3016-T, Mechanical and Aerospace Engineering, Princeton Univ., Princeton, NJ (2001).

${ }^{5}$ J.K. Ziemer and E.Y. Choueiri, "Scaling laws for electromagnetic pulsed plasma thrusters," Plasma Sources Sci. Technol., Vol. 10, No. 3 , 395-405 (2001).

${ }^{6}$ F.R. Poehlmann, M.A. Cappelli, and G.B. Reiker, "Current distribution measurements inside an electromagnetic plasma gun operated in gas-puff mode," Phys. Plasmas, Vol. 17, 123508 (2010).

${ }^{7}$ H. Sitaraman and L.L. Raja, "Magneto-hydrodynamics simulation study of deflagration mode in co-axial plasma accelerators," Phys. Plasmas, Vol. 21, 012104 (2014). 